\title{
Comparison of radical prostatectomy and external beam radiotherapy in high-risk prostate cancer
}

\author{
Seok-Joo Chun', Jin Ho Kim', Ja Hyeon Ku², Cheol Kwak², Eun Sik Lee², Suzy Kim,3 \\ ${ }^{1}$ Department of Radiation Oncology, Seoul National University College of Medicine, Seoul, Korea \\ ${ }^{2}$ Department of Urology, Seoul National University College of Medicine, Seoul, Korea \\ ${ }^{3}$ Department of Radiation Oncology, SMG-SNU Boramae Medical Center, Seoul, Korea
}

Received: May 7, 2021

Revised: May 28, 2021

Accepted: June 1, 2021

Correspondence:

Suzy Kim

Department of Radiation Oncology, SMG-SNU Boramae Medical Center, 20 Boramae-ro 5-gil, Dongjak-gu, Seoul 07061, Korea

Tel: +82-2-870-1691

E-mail: suzy101@snu.ac.kr

ORCID:

https://orcid.org/0000-0001-7161-8340
Purpose: We evaluated clinical outcomes of high-risk prostate cancer patients receiving external beam radiotherapy (EBRT) or radical prostatectomy $(\mathrm{RP})$.

Materials and Methods: Patients were classified as high-risk prostate cancer and received definitive treatment between 2005 and 2015. Patients with previous pelvic radiotherapy, positive lymph node or distant metastasis were excluded. The primary outcomes were prostate cancer-specific survival (PCSS) and distant metastasis-free survival (DMFS).

Results: Of 583 patients met the inclusion criteria (77 EBRT and 506 RP), the estimated 10-year PCSS was $97.0 \%$ in the RP and $95.9 \%$ in the EBRT ( $p=0.770$ ). No significant difference was seen in the DMFS ( $p=0.540$ ), whereas there was a trend in favor of RP over EBRT in overall survival (OS) ( $p=$ 0.068). Propensity score matching analysis with confounding variables was done, with 183 patients (66 EBRT and 117 RP) were included. No significant difference in DMFS, PCSS or OS was found.

Conclusion: Our data demonstrated similar oncologic PCSS, OS, and DMFS outcomes between EBRT and RP patients.

Keywords: Radiotherapy, Prostatectomy, Prostatic neoplasms

\section{Introduction}

A landmark, randomized, controlled study comparing radical prostatectomy (RP) and external beam radiotherapy (EBRT) in low-risk prostate cancer patients revealed similar oncologic outcomes between these two modalities [1]. Another randomized controlled trial comparing RP and watchful waiting reported a 2.9-year increase in life expectancy in patients that underwent RP [2]. However, these trials were based on low-risk prostate cancer and most patients had an initial prostate-specific antigen (PSA) less than 20 $\mathrm{ng} / \mathrm{mL}$ and clinical T1 or T2 disease.

Meanwhile, high-risk prostate cancer patients, defined as high initial PSA ( $\geq 20 \mathrm{ng} / \mathrm{mL}$ ), Gleason score $(\geq 8)$, or clinical T stage ( $\geq 3$ a) by the National Comprehensive Cancer Network (NCCN) have been widely accepted as aggressive tumors compared to low and intermediate-risk prostate cancers [3]. However, no large randomized trial has been reported on high-risk prostate diseases. Thus, the optimal local treatment for high-risk prostate cancer is still unknown. Several retrospective studies and meta-analysis have been published and demonstrated better oncologic survival of patients who received RP than those who were treated with EBRT [46]. However, recent studies demonstrated similar oncologic outcomes between RP and EBRT in patients with Gleason score 9-10 disease $[7,8]$. Currently, most guidelines recommend RP or EBRT with the addition of androgen deprivation therapy (ADT) as standard of care for locally advanced prostate cancer [3,9].

This study aimed to compare the prostate cancer-specific survival (PCSS), overall survival (OS), and distant metastasis-free survival (DMFS) between high-risk prostate cancer patients treated with RP or EBRT. Also, as very high-risk prostate cancer are closely related 
with poor prognosis [10,11], we aimed to compare oncologic outcome of RP and EBRT in very high-risk group.

\section{Materials and Methods}

The data on patients who were pathologically diagnosed with prostate cancer from 2005 to 2015 were retrospectively collected. All patients had at least one high-risk feature according to the NCCN guidelines (initial PSA $\geq 20 \mathrm{ng} / \mathrm{mL}$, Gleason score $\geq 8$, or clinical T stage $\geq 3$ a). Before treatment, all patients had transrectal prostate ultrasonographic biopsies and record of the number of biopsy cores and positive cores. The patients had undergone either RP or EBRT for definitive purposes. Patients who had pelvic radiotherapy before the diagnosis of prostate cancer were excluded. Patients with suspicious node metastasis or distant metastasis were also excluded. All patients had a minimum follow-up of 3 years. This study was approved by the Health Institutional Review Board of Seoul National University Hospital (No. H-1904-128-1028).

The patients were classified into the EBRT or RP groups. Local definitive treatment options were given to the patients in the outpatient urology clinic. Generally, relatively younger patients and those with fewer comorbidities were offered RP. Both robotic surgery and open retropubic prostatectomy were included in the RP group. Pelvic lymphadenectomy was done in half of the cases, at the preference of the urologist. In the presence of high recurrence indicators, such as positive surgical resections margins or lymph node metastasis, adjuvant radiotherapy or ADT was offered post-surgery. Adjuvant treatment was defined as the initiation of treatment within 6 months after surgery with PSA $<0.2 \mathrm{ng} / \mathrm{mL}$. Treatment intervals longer than 6 months or PSA levels greater than $0.2 \mathrm{ng} / \mathrm{mL}$ after surgery was considered to be salvage settings.

For definitive EBRT, most of the patients received ADT before or concurrently with EBRT. Most of the patients received intensity-modulated radiotherapy (IMRT). Before 2015, the patients received conventional fractionated radiotherapy, with a median dose of $81 \mathrm{~Gy}$ in 45 fractions. After the publication of randomized controlled trials of hypofractionation [12,13], 70 Gy in 28 fractions were commonly used. Clinical target volume (CTV) was usually confined to the prostate and seminal vesicles, often excluding pelvic lymph nodes. Planning target volume included CTV plus $5 \mathrm{~mm}$ to cover uncertainties during radiotherapy. Prostate brachytherapy was not performed in our institution.

Follow-up was usually done every 3 months until 2 years, then every 6 months until 5 years and annually thereafter. PSA was acquired at every follow-up. In the case of biochemical failure, pelvic magnetic resonance imaging and bone scans were done to check locoregional recurrence or bone metastasis. RP patients with bio- chemical failure were offered salvage ADT alone or salvage ADT followed by radiotherapy, whereas the EBRT patients received ADT alone. Salvage EBRT was given in a median dose of $70.2 \mathrm{~Gy}$ in 39 fractions to the prostate fossa. Pelvic lymph node irradiation was not routinely performed. If there was a failure after salvage treatment, chemotherapy or second-line ADT was offered until disease progression.

Biochemical failure has multiple definitions and they have changed during the treatment era. Also, different definitions of biochemical failure in RP and EBRT make it difficult to directly compare the outcomes of RP and EBRT. Thus, in this study, the endpoints were focused on PCSS, OS, and DMFS. The base of follow-up defined as the day of RP or the first day of EBRT or ADT. Survival length was calculated as the period from the start of follow-up to the event date. Prostate-specific death was classified by the main investigators as death after evident signs of distant metastasis or non-metastatic castration-resistant prostate cancer.

To compare the patient characteristics between the RP and EBRT groups, chi-squared analysis was used for categorical data, but for a small sample size, Fisher exact test was done. For comparison of survival data, the method by Kaplan-Meier and the Mantel-Cox logrank test were used. Multivariate analysis was done based on Cox proportional hazards regression model for DMFS and OS. For PCSS, Fine and Gray proportional hazard regression model [14] was used assuming non prostate-specific death as competitive factor. Factors which had $p$-value less than 0.1 in univariate analysis were chosen for multivariate analysis. Factors with $p$-values less than 0.05 were defined as statistically significant. To reduce selection bias caused by confounding factors, propensity score matching was used. Propensity scores were calculated by a logistic regression model using factors with a significant effect on OS or PCSS. The nearest neighbor matching algorithm was used to select best control matches of $1: 2$ ratio with a caliper size of 0.25 . Matchlt package in $R$ was used for propensity score matching analysis [15]. All the analyses were performed using R 3.5.2 (http://www.r-project.org/).

\section{Results}

A total of 583 patients were analyzed. Seventy-seven patients received EBRT and 506 received RP. The baseline characteristics are described in Table 1. Patients with EBRT were significantly older, had higher initial PSA levels, and higher rates of core biopsies $\geq 5$ with Gleason scores of 8-10. The median dose of EBRT was 81 Gy given in 45 fractions. Three patients had relatively lower doses of $70.2 \mathrm{~Gy}$ in 39 fractions, whereas others received 75 Gy or more. All except three had IMRT. Sixty-seven patients (87.0\%) had ADT before or concurrently with EBRT. The median length of ADT was 25.0 months 
Table 1. Patient characteristics of all patients and matched cohorts

\begin{tabular}{|c|c|c|c|c|c|c|}
\hline \multirow{2}{*}{ Variable } & \multicolumn{3}{|c|}{ All patients } & \multicolumn{3}{|c|}{ Matched cohorts } \\
\hline & EBRT $(n=77)$ & $\mathrm{RP}(n=506)$ & p-value & $\operatorname{EBRT}(n=66)$ & $\operatorname{RP}(n=117)$ & $p$-value \\
\hline Age at diagnosis (yr) & $73.1 \pm 7.6$ & $66.9 \pm 7.0$ & $<0.001^{*}$ & $72.0 \pm 7.4$ & $71.9 \pm 6.0$ & 0.888 \\
\hline Gleason score (sum) & & & 0.305 & & & 0.841 \\
\hline $6-8$ & $61(79.2)$ & $428(84.6)$ & & $52(78.8)$ & 95 (81.2) & \\
\hline $9-10$ & $16(20.8)$ & $78(15.4)$ & & $14(21.2)$ & $22(18.8)$ & \\
\hline Initial PSA (ng/mL) & $40.3 \pm 50.3$ & $18.9 \pm 18.5$ & $<0.001^{*}$ & $33.7 \pm 35.8$ & $27.4 \pm 25.4$ & 0.210 \\
\hline Clinical stage & & & 0.087 & & & 0.896 \\
\hline T1-T3a & $50(64.9)$ & $379(74.9)$ & & 46 (69.7) & $84(71.8)$ & \\
\hline $\mathrm{T} 3 \mathrm{~b}-\mathrm{T} 4$ & $27(35.1)$ & $127(25.1)$ & & $20(30.3)$ & $33(28.2)$ & \\
\hline Core biopsy $\geq 5$ with Gleason score 8-10 & & & $0.001^{*}$ & & & 0.999 \\
\hline No & $54(70.1)$ & $435(86.0)$ & & 47 (71.2) & $84(71.8)$ & \\
\hline Yes & $23(29.9)$ & $71(14.0)$ & & $19(28.8)$ & $33(28.2)$ & \\
\hline Year of treatment & & & 0.588 & & & 0.571 \\
\hline $2005-2009$ & $22(28.6)$ & $164(32.4)$ & & $18(27.3)$ & $38(32.5)$ & \\
\hline 2010-2015 & $55(71.4)$ & $342(67.6)$ & & $48(72.7)$ & $79(67.5)$ & \\
\hline Charlson Comorbidity Index & & & 0.052 & & & 0.733 \\
\hline 0 & $41(53.2)$ & $331(65.4)$ & & $37(56.1)$ & $37(56.1)$ & \\
\hline$>0$ & $36(46.8)$ & $175(34.6)$ & & $29(43.9)$ & $29(43.9)$ & \\
\hline
\end{tabular}

Values are presented as mean \pm standard deviation or number of patients $(\%)$.

EBRT, external beam radiotherapy; $R P$, radical prostatectomy; PSA, prostate-specific antigen.

${ }^{*} p<0.05$.

(range, 4.2 to 48.5 months). Surgery was usually done by an open technique (61.1\%). Adverse pathologic features were found in many patients after surgery; $20.1 \%$ of the patients had positive pelvic lymph nodes, even though no signs of lymph node metastasis were seen in preoperative imaging. Also, about half of the patients had positive surgical resection margins and $78.9 \%$ had at least one finding, such as perineural invasion, lymphatic invasion or venous invasion. After surgery, adjuvant treatment was given to $16.2 \%$ of the patients, most of whom received ADT alone. Table 2 summarizes the treatment profiles and pathologic features in each arm.

After a median follow-up 6.25 years (range, 3.2 to 14.0 years), 38 patients were diagnosed with distant metastasis; five in the EBRT group (6.5\%) and 33 in the RP group (6.5\%). Of them, 30 patients had bone metastasis as the first site of distant metastasis, 6 as distant lymph node metastasis, 1 lung and 1 brain metastasis. The median time to distant metastasis was 4.18 years (range, 0.1 to 11.1 years). The estimated 5-year and 10-year DMFS were $98.7 \%$ and $93.7 \%$ in the EBRT and $95.7 \%$ and $90.4 \%$ in the RP group, respectively. Fig. 1A shows the Kaplan-Meier curve of DMFS in each $\operatorname{arm}(p=0.540)$. In univariate analysis, Gleason score $\geq 9$, clinical $T$ stage $\geq 3 b$, and core biopsy $\geq 5$ with Gleason score 8-10 were associated with significantly worse prognoses.

There were 65 deaths in the total cohort, with 16 prostate-specific deaths. Fourteen of the patients (18.1\%) who received EBRT died, three (3.9\%) due to prostate cancer. In the RP arm, 51 pa- tients (9.8\%) died, 13 (2.5\%) from prostate cancer-related causes. The estimated 5-year and 10-year OS were 94.6\% and $77.0 \%$ in the EBRT arm and $97.7 \%$ and $85.8 \%$ in the RP arm, respectively. Although there was a trend toward an increase in any cause of death by EBRT ( $p=0.071$ ), this difference was insignificant in multivariate analysis ( $p=0.785$ ). No significant difference in PCSS was found between the two arms (5-year and 10-year PCSS, 98.7\% and $96.0 \%$ in the EBRT group; 99.2\% and 97\% in the RP group, respectively). Fig. $1 \mathrm{~B}$ and $1 \mathrm{C}$ shows the survival curves for $\mathrm{OS}$ and PCSS in the EBRT and RP patients. In variables related to overall survival, higher age, advanced clinical T stage, higher Charlson Comorbidity Index, and treatment modality were significantly associated with poor survival. After multivariate analysis, older age ( $p=$ $0.001)$ and advanced T stage ( $p=0.008)$ were selected as significant variables for overall survival. For PCSS, Gleason score $\geq 9$, clinical T stage $\geq 3 b$, and core biopsy $\geq 5$ with Gleason score 8-10 were associated with poor prognoses in univariate analysis. Gleason score and clinical T stage were still identified as prognostic factors for PCSS after multivariate analysis. Table 3 shows the multivariate analysis results for DMFS, OS, and PCSS.

Clinical T stage $\geq 3 b$, Gleason score $\geq 9$, and core biopsy $\geq 5$ with Gleason score 8-10 were very closely associated with poor prognoses for DMFS or PCSS. These variables are also known as risk factors for very high-risk (VHR) prostate cancer in the NCCN guidelines. Not only was there a significant difference between VHR 
Table 2. Treatment and pathologic details of patients with EBRT and RP

\begin{tabular}{|c|c|c|}
\hline & $\operatorname{EBRT}(\mathrm{n}=77)$ & $\mathrm{RP}(\mathrm{n}=506)$ \\
\hline \multicolumn{3}{|l|}{ ADT } \\
\hline Use of ADT & $67(87.0)$ & - \\
\hline Duration (m) & $25.0(4.2-48.5)$ & - \\
\hline \multicolumn{3}{|l|}{ EBRT profile } \\
\hline EBRT dose (Gy) & $81.0(70.0-81.0)$ & - \\
\hline EBRT fraction & $45(28-45)$ & - \\
\hline \multicolumn{3}{|l|}{ EBRT technique } \\
\hline 3D-CRT & $3(3.9)$ & - \\
\hline IMRT & $34(44.2)$ & - \\
\hline VMAT & 40 (51.9) & - \\
\hline \multicolumn{3}{|l|}{ EBRT field } \\
\hline Prostate only & $73(94.8)$ & - \\
\hline Prostate + pelvic lymph node & $4(5.2)$ & - \\
\hline \multicolumn{3}{|l|}{ Surgery technique } \\
\hline Open & - & $309(61.1)$ \\
\hline Robotic/laparoscopic & - & $197(38.9)$ \\
\hline \multicolumn{3}{|l|}{ Pelvic node dissection } \\
\hline Yes & - & $313(61.9)$ \\
\hline No & - & $193(38.1)$ \\
\hline \multicolumn{3}{|l|}{ Pathologic adverse feature } \\
\hline $\mathrm{T} 3 \mathrm{~b} / \mathrm{T} 4$ & - & $150(29.6)$ \\
\hline Positive lymph nodes ${ }^{a)}$ & - & $63(20.1)^{a)}$ \\
\hline Positive resection margin & - & $274(54.2)$ \\
\hline Positive PNI/LVI & - & 399 (78.9) \\
\hline \multicolumn{3}{|l|}{ Adjuvant treatment } \\
\hline Total & - & $82(16.2)$ \\
\hline EBRT alone & - & $15(3.0)$ \\
\hline $\mathrm{EBRT}+\mathrm{ADT}$ & - & $16(3.2)$ \\
\hline ADT alone & - & $51(10.1)$ \\
\hline \multicolumn{3}{|l|}{ Salvage treatment } \\
\hline Total & 14 (18.2) & $286(56.5)$ \\
\hline EBRT alone & 0 & $18(3.6)$ \\
\hline $\mathrm{EBRT}+\mathrm{ADT}$ & 0 & 99 (19.6) \\
\hline ADT alone & 13 (16.9) & $155(30.6)$ \\
\hline Chemotherapy & $1(1.3)$ & 0 \\
\hline Observation & 0 & $14(2.8)$ \\
\hline
\end{tabular}

Values are presented as number of patients (\%).

EBRT, external beam radiotherapy; $R P$, radical prostatectomy; $A D T$, androgen deprivation therapy; 3D-CRT, three-dimensional conformal radiotherapy IMRT, intensity-modulated radiotherapy; VMAT, volumetric arc therapy; PNI, perineural invasion; LVI, lymphovascular invasion.

${ }^{a)}$ Positive lymph nodes were calculated in patients who received pelvic node dissection.

prostate cancer and non-VHR prostate cancer $(p<0.001)$, patients with multiple VHR features had poorer PCSS compared to patients with one VHR feature or non-VHR. Fig. 2 shows the survival difference between patients with VHR features and non-VHR. No significant difference in DMFS or PCSS between the treatment modali- ties was seen in the VHR prostate cancer patients.

Clinical variables that had a clinical impact on DMFS, OS, and PCSS were selected for propensity score matching. As a result, age ( $\geq 70$ years), Gleason score $(\geq 9)$, clinical T stage ( $\geq 3 b$ ), and core biopsy $\geq 5$ with Gleason score 8-10, and Charlson Comorbidity Index were included in the model. After matching, a total of 183 patients were selected, with 66 in EBRT and 117 in RP arm. There were no statistical differences in age at diagnosis, Gleason score, initial PSA level, clinical T stage, core biopsy $\geq 5$ with Gleason score 8-10, year of treatment or Charlson Comorbidity Index. Table 1 shows the clinical characteristics of the matched cohorts. No significant differences in DMFS, OS, and PCSS were found between the EBRT and RP arms in the matched cohort (Fig. 3).

Salvage treatment was offered to 300 patients (50.5\%), with more in the RP arms ( $n=286 ; 56.5 \%)$ than in the EBRT arm ( $\mathrm{n}=$ $14 ; 18.2 \% ; p<0.001)$. Most patients in the RP arm received salvage EBRT combined with ADT or ADT alone, whereas most patients in the EBRT arm received salvage ADT alone. Salvage treatment was more often required in patients with Gleason scores $\geq 9$ ( $p=$ 0.013 ), clinical T stage $\geq 3 b$ ( $p=0.079$ ), core biopsy $\geq 5$ with Gleason score 8-10 ( $<<0.001)$, and $\operatorname{RP}(p<0.001)$. After multivariate analysis of those four factors, core biopsy $\geq 5$ with Gleason score 8-10 and RP were associated with shorter salvage treatment-free survival (Table 4). After propensity matching, 72 patients (61.5\%) in $\mathrm{RP}$ arm received salvage treatment-41 received ADT alone, $28 \mathrm{RT}$ + ADT, and 3 RT alone. While 13 patients (19.7\%) in RT arm received salvage treatment-11 with ADT alone, 1 ADT with salvage operation for para-aortic lymph node, and 1 chemotherapy.

We found 21 grade 2 toxicities, 16 (3.2\%) in RP group and 5 $(6.5 \%)$ in EBRT group. Mainly, there were hematuria or hematochezia in EBRT patients, while there were more cases of erectile dysfunction in RP arm. No life-threatening toxicities were reported.

\section{Discussion and Conclusion}

Our study demonstrated similar oncologic PCSS and DMFS outcomes between the EBRT and RP arms. In unmatched cohorts, there was a significant difference in OS. However, because there was a tendency for patients with older age, higher initial PSA levels, higher clinical T stages, and more comorbidities to receive EBRT, it is reasonable that the difference in OS was due to these confounding factors, not the treatment modality. This hypothesis is supported by the fact that no OS difference was found between two arms after propensity score matching.

Several studies on local treatment modalities in high-risk prostate cancer have been conducted. In 2014, Petrelli et al. [5] reported a meta-analysis of 17 studies and concluded that surgery was 


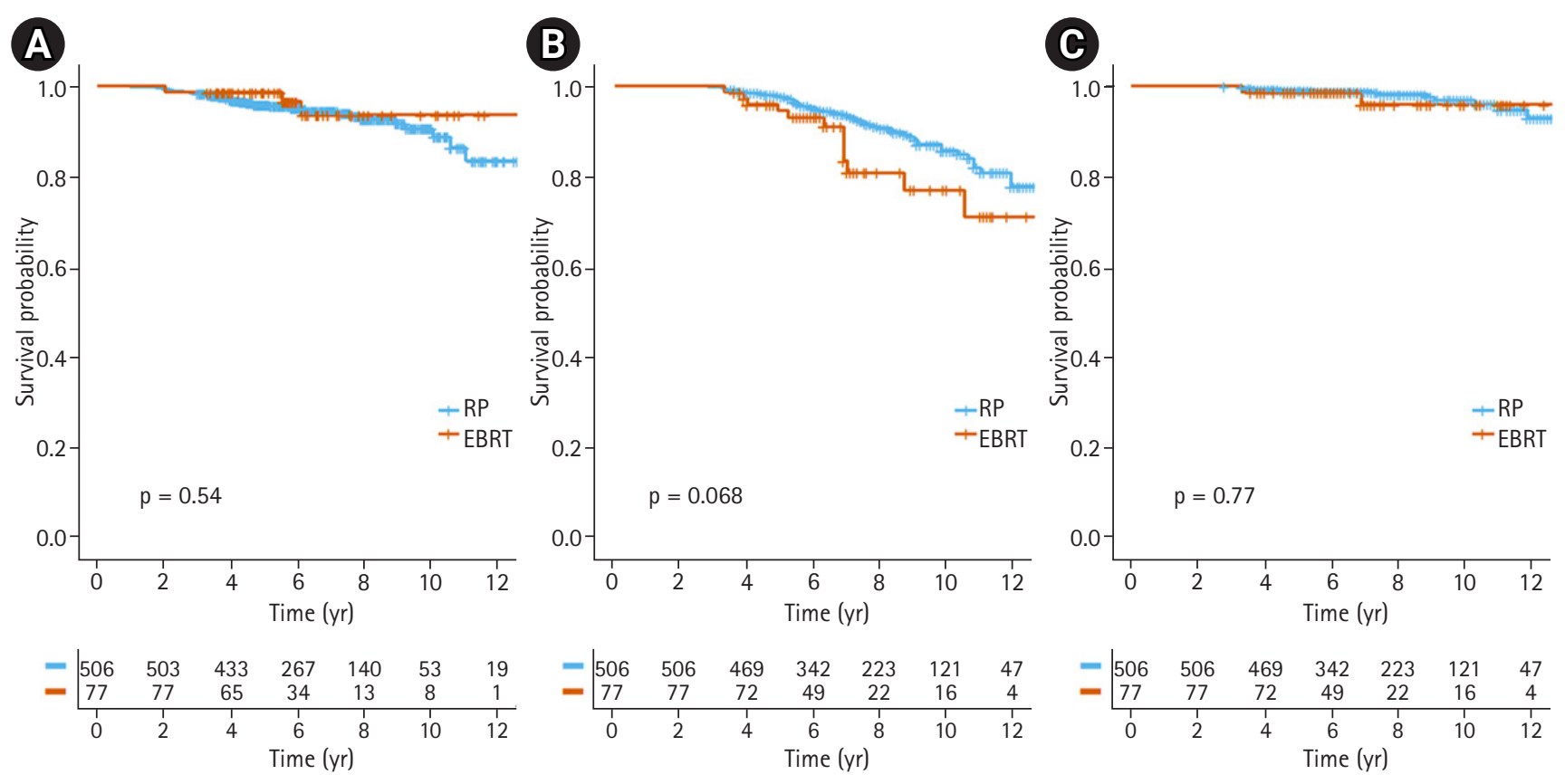

Fig. 1. Kaplan-Meier survival curves of (A) distant metastasis-free survival, (B) overall survival, and (C) prostate cancer-specific survival for patients with radical prostatectomy (RP) and external beam radiotherapy (EBRT).

Table 3. Univariate and multivariate analysis of factors for distant metastasis, death from any cause, and death from prostate cancer

\begin{tabular}{|c|c|c|c|c|c|c|c|c|c|c|c|c|}
\hline & \multicolumn{4}{|c|}{ Distant metastasis } & \multicolumn{4}{|c|}{ Death from any cause } & \multicolumn{4}{|c|}{ Death from prostate cancer } \\
\hline & \multicolumn{2}{|c|}{ Univariate } & \multicolumn{2}{|c|}{ Multivariate } & \multicolumn{2}{|c|}{ Univariate } & \multicolumn{2}{|c|}{ Multivariate } & \multicolumn{2}{|c|}{ Univariate } & \multicolumn{2}{|c|}{ Multivariate } \\
\hline & $\mathrm{HR}$ & $p$-value & $\mathrm{HR}$ & $p$-value & $\mathrm{HR}$ & p-value & $\mathrm{HR}$ & p-value & $\mathrm{HR}$ & p-value & HR & $p$-value \\
\hline Age at diagnosis ( $>70$ vs. $\leq 70 \mathrm{yr}$ ) & 1.19 & 0.617 & - & - & 2.69 & $<0.001^{*}$ & 2.44 & $0.001^{*}$ & 2.04 & 0.188 & - & - \\
\hline Gleason score (sum 9-10 vs. 6-8) & 5.24 & $<0.001^{*}$ & 2.39 & $0.04^{*}$ & 1.50 & 0.180 & - & - & 6.66 & $<0.001^{*}$ & 4.26 & $0.01^{*}$ \\
\hline Initial PSA (> 20 vs. $\leq 20 \mathrm{ng} / \mathrm{mL}$ ) & 1.12 & 0.748 & - & - & 1.04 & 0.895 & - & - & 0.65 & 0.460 & - & - \\
\hline Clinical stage (cT3b-4 vs. cT1-3a) & 2.60 & $0.006^{*}$ & 1.86 & 0.08 & 2.05 & $0.005^{*}$ & 1.98 & $0.008^{*}$ & 9.32 & $0.001^{*}$ & 7.25 & $<0.01^{*}$ \\
\hline $\begin{array}{l}\text { Core biopsy } \geq 5 \text { with Gleason score 8-10 } \\
\text { (yes vs. no) }\end{array}$ & 5.96 & $<0.001^{*}$ & 3.38 & $0.00^{*}$ & 1.66 & 0.104 & - & - & 4.56 & $0.005^{*}$ & 1.55 & 0.43 \\
\hline Year of treatment (2010-2015 vs. 2005-2009) & 0.53 & 0.108 & - & - & 0.74 & 0.325 & - & - & 0.41 & 0.230 & - & - \\
\hline Charlson Comorbidity Index (>0 vs. 0) & 0.88 & 0.733 & - & - & 1.83 & $0.018^{*}$ & 1.62 & 0.060 & 1.46 & 0.484 & - & - \\
\hline Treatment modality (RP vs. EBRT) & 1.44 & 0.541 & - & - & 0.55 & 0.071 & 0.91 & 0.785 & 0.79 & 0.766 & - & - \\
\hline
\end{tabular}

$H R$, hazard ratio; PSA, prostate-specific antigen; RP, radical prostatectomy; EBRT, external beam radiotherapy. ${ }^{*} p<0.05$.

associated with better PCSS and OS. Also, multiple retrospective studies have favored RP over EBRT $[4,6,16]$. However, it should be noted that these studies were based on patients in the 20th century and the early 2000s. In EBRT, the introduction of IMRT has presented the possibility of dose escalation for prostate cancer, while reducing doses to the bladder and rectum [17-19]. Along with IMRT, the combined use of ADT with EBRT has become standard treatment for advanced prostate cancer $[20,21]$. Due to these technical improvements and new treatment strategies, treatment outcomes have improved over time. Robinson et al. [22] demonstrated that treatment outcomes have improved by year of treatment, especially in the EBRT arm. They also reported smaller treatment dif- ferences in the EBRT and RP arms in patients who were treated after 2008. Recent studies have also shown similar trends [7,8,22]. Kishan et al. [7] reported that EBRT combined with brachytherapy was associated with better survival outcomes compared to EBRT alone or RP, whereas similar survival data were shown between EBRT alone and RP. Based on recent data and the results from this study, EBRT and RP seemed to have similar effects on controlling high-risk prostate cancer in the current era.

Biochemical failure was not reported in this paper due to different definitions of the two modalities. However, the percentage of salvage treatment was much higher in the RP arm compared to the EBRT arm. The high rate of salvage treatment in the RP group 

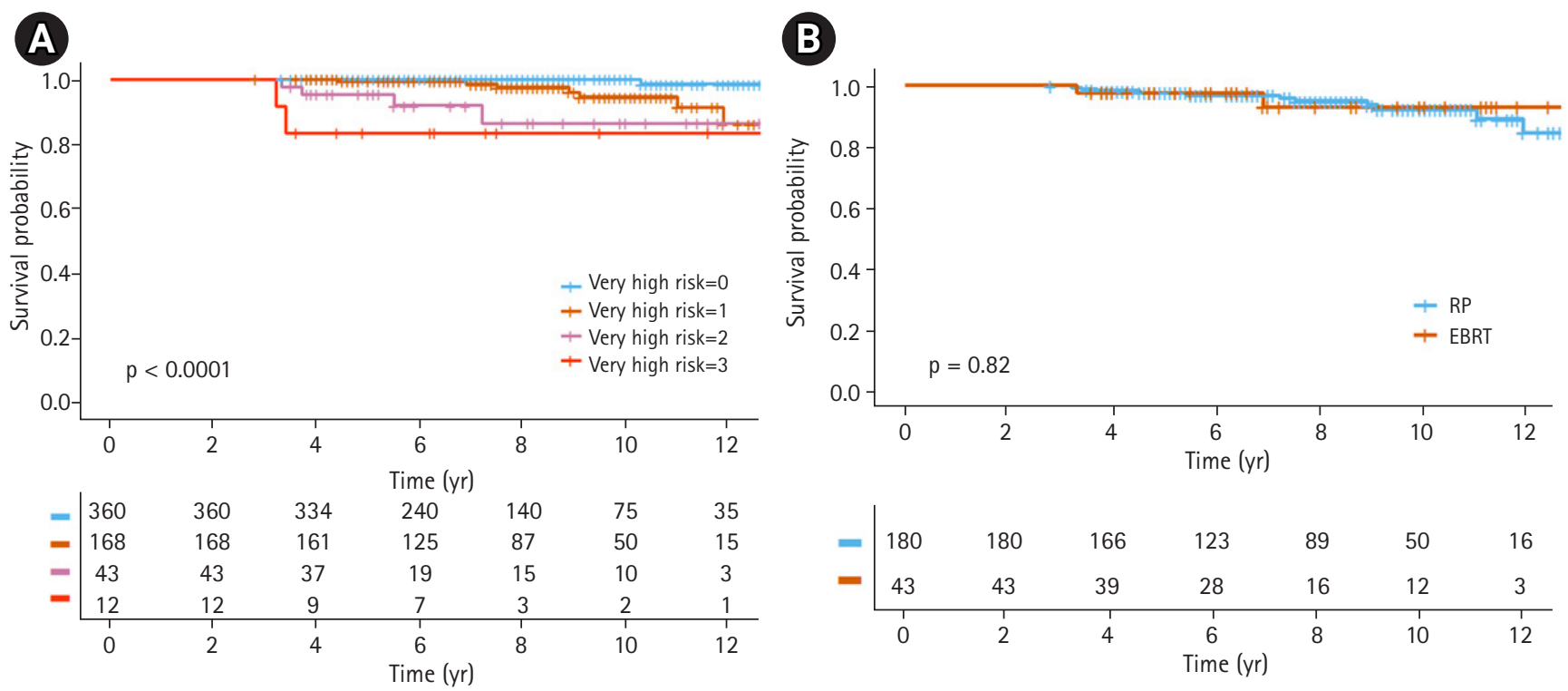

Fig. 2. Kaplan-Meier survival curves of (A) prostate cancer-specific survival (PCSS) for patients according to number of very high-risk features and (B) PCSS for external beam radiotherapy (EBRT) and radical prostatectomy (RP) in very high-risk prostate cancer.

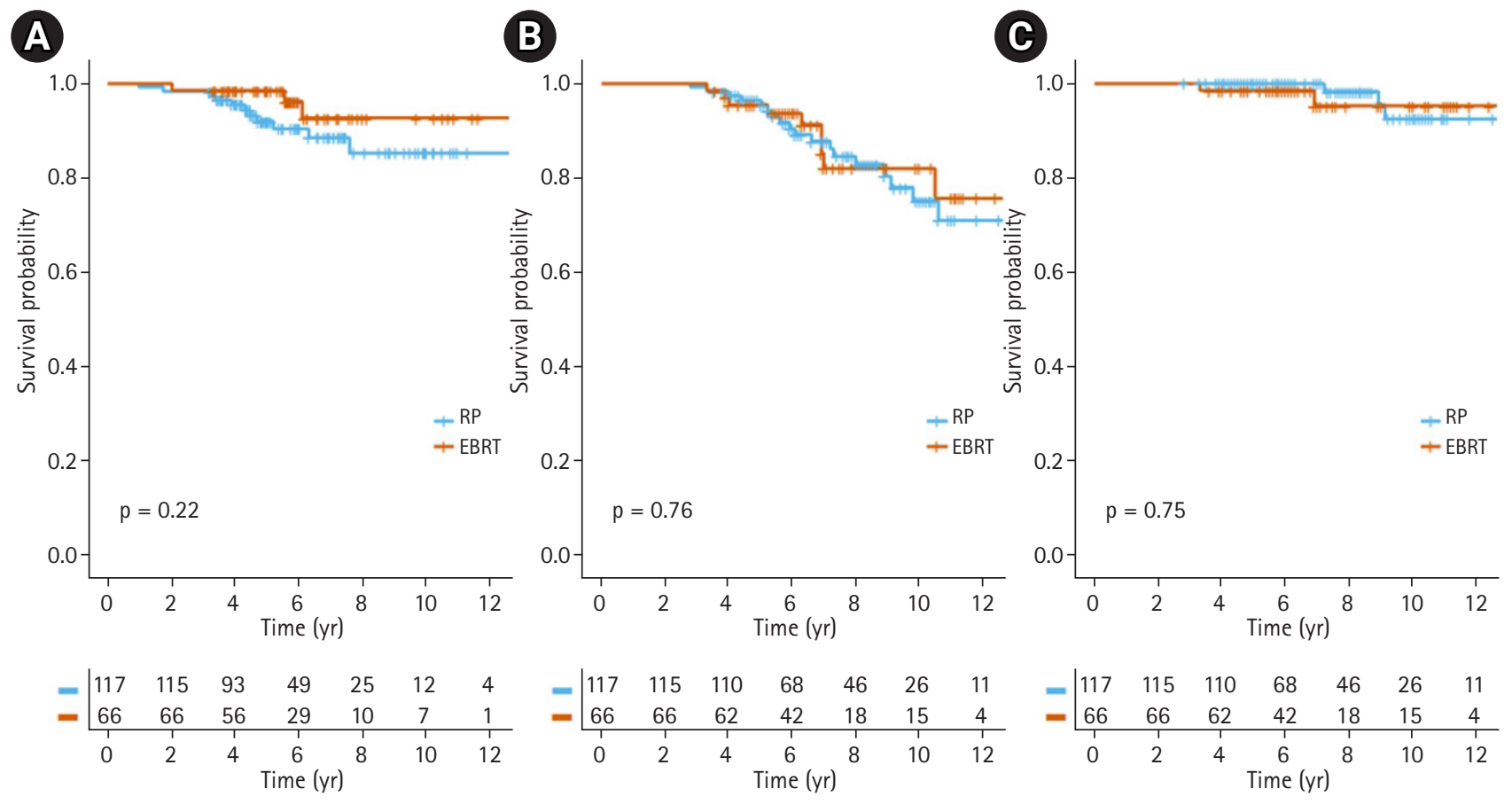

Fig. 3. Kaplan-Meier survival curves of (A) distant metastasis-free survival, (B) overall survival, and (C) prostate cancer-specific survival for patients with radical prostatectomy $(\mathrm{RP})$ and external beam radiotherapy (EBRT) after propensity score matching.

might be attributable to several factors. First, positive surgical resection margins were reported in 54.2\% of the patients and positive surgical resection margins are closely associated with higher progression and survival rates $[23,24]$. Advanced clinical T stage and the high rate $(78.9 \%)$ of positive perineural invasion or lymphovascular invasion would have made complete surgical removal even harder. Second, salvage EBRT could be effectively given after
$\mathrm{RP}$, whereas salvage RP is not commonly performed after an initial EBRT. Our study also showed that no salvage surgery was performed on patients in the EBRT arm, whereas 40.9\% (117/286) of the patients received EBRT alone or EBRT combined with ADT as salvage treatment in the RP arm. Third, the definition of adjuvant treatment was strictly applied in this study. Since adjuvant treatment was defined as both low PSA levels ( $<0.2 \mathrm{ng} / \mathrm{mL}$ for RP) and 
Table 4. Multivariate analysis of factors for salvage treatment event

\begin{tabular}{lcc}
\hline & \multicolumn{2}{c}{ Salvage treatment event } \\
\hline Age at diagnosis ( $>$ 70 vs. $\leq 70$ yr) & HR (95\% Cl) & - \\
Gleason score (sum 9-10 vs. 6-8) & - & 0.841 \\
Initial PSA (> 20 vs. $\leq 20 \mathrm{ng} / \mathrm{mL}$ ) & - & - \\
Clinical stage (cT3b-4 vs. cT1-3a) & $1.29(1.00-1.66)$ & 0.052 \\
Core biopsy $\geq 5$ with Gleason score 8-10 (yes vs. no) & $2.16(1.54-3.03)$ & $<0.001$ \\
Year of treatment (2010-2015 vs. 2005-2009) & - & - \\
Charlson Comorbidity Index (>0 vs. 0) & - & - \\
Treatment modality (RP vs. EBRT) & $4.95(2.87-8.53)$ & $<0.001$
\end{tabular}

$\mathrm{HR}$, hazard ratio; $\mathrm{Cl}$, confidence interval; PSA, prostate specific antigen; $\mathrm{RP}$, radical prostatectomy; EBRT, external beam radiotherapy.

treatment intervals of less than six months after treatment, patients who received treatment after increased postoperative PSA levels were classified as receiving salvage treatment. Despite these factors, salvage treatments were given more often to patients in the RP arm, even after propensity score matching.

It is well known that VHR prostate cancer patients have more aggressive features compared to non-VHR prostate cancer patients $[10,11]$. Our study also demonstrated both worse PCSS and DMFS in VHR prostate cancer patients. Notably, no survival difference was seen between the RP and EBRT arms. However, relatively poorer prognosis compared to high-risk prostate cancer without VHR features might lead to intensive treatment. A randomized controlled trial adding brachytherapy to EBRT plus ADT demonstrated improved biochemical progression-free survival in high-risk prostate cancer patients [25]. Consistent with that study, this study suggested that adding brachytherapy and ADT to EBRT might be an optimal approach to treating VHR prostate cancer. However, clinical trials are needed to confirm the results.

One limitation of this study was its retrospective design. Although we used propensity score matching to reduce selection bias, we acknowledge that there may have been other factors that were not adjusted for that may have affected the survival rates. Also, toxicity profiles were not collected because half of the patients did not complete questionnaires on patient-reported outcomes. Without these data, proper comparison could not be made. However, previous multiple, randomized controlled studies have shown distinctly different patterns in the quality of life of patients who received EBRT or RP, which can also apply to our study [26,27]. A strength of our study is that it was a large single-institution study of treatments with modern techniques, mainly EBRT combined with ADT and IMRT. Also, this study was based on a single race, which is known to be a cofounding factor for prostate cancer. Hopefully, SPCG-15, a randomized trial investigating the clinical effectiveness of RP and EBRT plus ADT that is currently underway, will provide answers to this long-debated question [28].

\section{Conflict of Interest}

No potential conflict of interest relevant to this article was reported.

\section{References}

1. Hamdy FC, Donovan JL, Lane JA, et al. 10-year outcomes after monitoring, surgery, or radiotherapy for localized prostate cancer. N Engl J Med 2016;375:1415-24.

2. Bill-Axelson A, Holmberg L, Garmo H, et al. Radical prostatectomy or watchful waiting in prostate cancer: 29-year follow-up. N Engl J Med 2018;379:2319-29.

3. Mohler JL, Antonarakis ES, Armstrong AJ, et al. Prostate cancer, version 2.2019, NCCN clinical practice guidelines in oncology. J Natl Compr Canc Netw 2019;17:479-505.

4. Hoffman RM, Koyama T, Fan KH, et al. Mortality after radical prostatectomy or external beam radiotherapy for localized prostate cancer. J Natl Cancer Inst 2013;105:711-8.

5. Petrelli F, Vavassori I, Coinu A, Borgonovo K, Sarti E, Barni S. Radical prostatectomy or radiotherapy in high-risk prostate cancer: a systematic review and metaanalysis. Clin Genitourin Cancer 2014;12:215-24.

6. Sooriakumaran P, Nyberg T, Akre O, et al. Comparative effectiveness of radical prostatectomy and radiotherapy in prostate cancer: observational study of mortality outcomes. BMJ 2014; 348:g1502.

7. Kishan $A U$, Cook RR, Ciezki JP, et al. Radical prostatectomy, external beam radiotherapy, or external beam radiotherapy with brachytherapy boost and disease progression and mortality in patients with Gleason score 9-10 prostate cancer. JAMA 2018; 319:896-905. 
8. Tilki $\mathrm{D}$, Chen $\mathrm{MH}, \mathrm{Wu}$ J, et al. Surgery vs radiotherapy in the management of biopsy Gleason score 9-10 prostate cancer and the risk of mortality. JAMA Oncol 2019;5:213-20.

9. Parker C, Gillessen $S_{1}$ Heidenreich $A_{1}$ Horwich $A_{\text {; }}$ ESMO Guidelines Committee. Cancer of the prostate: ESMO Clinical Practice Guidelines for diagnosis, treatment and follow-up. Ann Oncol 2015;26 Suppl 5:v69-77.

10. Sundi D, Wang VM, Pierorazio PM, et al. Very-high-risk localized prostate cancer: definition and outcomes. Prostate Cancer Prostatic Dis 2014;17:57-63.

11. Narang AK, Gergis $C$, Robertson SP, et al. Very high-risk localized prostate cancer: outcomes following definitive radiation. Int J Radiat Oncol Biol Phys 2016;94:254-62.

12. Arcangeli G, Saracino B, Arcangeli S, et al. Moderate hypofractionation in high-risk, organ-confined prostate cancer: final results of a phase III randomized trial. J Clin Oncol 2017;35:1891-7.

13. Incrocci L, Wortel RC, Alemayehu WG, et al. Hypofractionated versus conventionally fractionated radiotherapy for patients with localised prostate cancer (HYPRO): final efficacy results from a randomised, multicentre, open-label, phase 3 trial. Lancet Oncol 2016;17:1061-9.

14. Fine JP, Gray RJ. A proportional hazards model for the subdistribution of a competing risk. J Am Stat Assoc 1999;94:496-509.

15. Gupta A, Madhavan MV, Sehgal K, et al. Extrapulmonary manifestations of COVID-19. Nat Med 2020;26:1017-32.

16. Sun $M$, Sammon JD, Becker A, et al. Radical prostatectomy vs radiotherapy vs observation among older patients with clinically localized prostate cancer: a comparative effectiveness evaluation. BJU Int 2014;113:200-8.

17. Zelefsky MJ, Fuks Z, Happersett L, et al. Clinical experience with intensity modulated radiation therapy (IMRT) in prostate cancer. Radiother Oncol 2000;55:241-9.

18. Al-Mamgani A, Heemsbergen WD, Peeters ST, Lebesque JV. Role of intensity-modulated radiotherapy in reducing toxicity in dose escalation for localized prostate cancer. Int J Radiat Oncol Biol Phys 2009;73:685-91.

19. Zelefsky MJ, Levin EJ, Hunt M, et al. Incidence of late rectal and urinary toxicities after three-dimensional conformal radiotherapy and intensity-modulated radiotherapy for localized prostate can- cer. Int J Radiat Oncol Biol Phys 2008;70:1124-9.

20. Bolla M, Collette L, Blank L, et al. Long-term results with immediate androgen suppression and external irradiation in patients with locally advanced prostate cancer (an EORTC study): a phase III randomised trial. Lancet 2002;360:103-6.

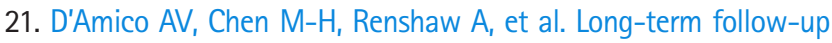
of a randomized trial of radiation with or without androgen deprivation therapy for localized prostate cancer. JAMA 2015; 314:1291-3.

22. Robinson D, Garmo H, Lissbrant IF, et al. Prostate cancer death after radiotherapy or radical prostatectomy: a nationwide population-based observational study. Eur Urol 2018;73:502-11.

23. Karakiewicz PI, Eastham JA, Graefen M, et al. Prognostic impact of positive surgical margins in surgically treated prostate cancer: multi-institutional assessment of 5831 patients. Urology 2005; 66:1245-50.

24. Grossfeld GD, Chang JJ, Broering JM, et al. Impact of positive surgical margins on prostate cancer recurrence and the use of secondary cancer treatment: data from the CaPSURE database. J Urol 2000;163:1171-7.

25. Morris WJ, Tyldesley S, Rodda S, et al. Androgen suppression combined with elective nodal and dose escalated radiation therapy (the ASCENDE-RT Trial): an analysis of survival endpoints for a randomized trial comparing a low-dose-rate brachytherapy boost to a dose-escalated external beam boost for high- and intermediate-risk prostate cancer. Int J Radiat Oncol Biol Phys 2017;98:275-85.

26. Chen RC, Basak R, Meyer AM, et al. Association between choice of radical prostatectomy, external beam radiotherapy, brachytherapy, or active surveillance and patient-reported quality of life among men with localized prostate cancer. JAMA 2017; 317:1141-50.

27. Donovan JL, Hamdy FC, Lane JA, et al. Patient-reported outcomes after monitoring, surgery, or radiotherapy for prostate cancer. $\mathrm{N}$ Engl J Med 2016;375:1425-37.

28. Stranne J, Brasso K, Brennhovd B, et al. SPCG-15: a prospective randomized study comparing primary radical prostatectomy and primary radiotherapy plus androgen deprivation therapy for locally advanced prostate cancer. Scand J Urol 2018;52:313-20. 